\title{
Lidil
}

Revue de linguistique et de didactique des langues

$48 \mid 2013$

L'émotion et l'apprentissage des langues

\section{Humour et apprentissage des langues : une typologie de séquences pédagogiques}

Joséphine Rémon

\section{OpenEdition}

Journals

Édition électronique

URL : http://journals.openedition.org/lidil/3317

DOI : 10.4000/lidil.3317

ISSN : 1960-6052

Éditeur

UGA Éditions/Université Grenoble Alpes

Édition imprimée

Date de publication : 1 novembre 2013

Pagination : 77-95

ISBN : $978-2-84310-260-8$

ISSN : $1146-6480$

Référence électronique

Joséphine Rémon, «Humour et apprentissage des langues : une typologie de séquences

pédagogiques », Lidil [En ligne], 48 | 2013, mis en ligne le 01 mai 2015, consulté le 19 avril 2019. URL

http://journals.openedition.org/lidil/3317; DOI : 10.4000/lidil.3317

(c) Lidil 


\title{
Humour et apprentissage des langues : une typologie de séquences pédagogiques
}

\author{
Joséphine Rémon*
}

\begin{abstract}
RÉSUMÉ
Pour aborder l'émotion en apprentissage des langues, nous interrogeons l'humour comme facilitateur face à des émotions inhibitrices en pratique de la langue, à travers des exemples de séquences pédagogiques. Nous proposons une typologie de ces séquences faisant intervenir l'humour, que celui-ci soit du fait de l'enseignant ou du fait de l'apprenant. Cette typologie nous permet de mettre en évidence la diversité que recouvre le recours à l'humour dans un contexte pédagogique. Dans une perspective où la production langagière est considérée comme prise de risque langagière, nous étudions la façon dont l'humour fait partie intégrante d'un scénario pédagogique ou au contraire fait irruption dans une séquence. L'humour convoque en effet des émotions variées, puisqu'il représente, selon les cas, une prise de risque pédagogique pour l'enseignant ou un risque de perte de face pour l'apprenant. Nous faisons l'hypothèse qu'une intégration de l'humour non menaçante contribue à la mise en place d'une communauté de confiance dans la classe de langue et permet d'encourager les émotions favorables à la prise de risque langagière.
\end{abstract}

\section{ABSTRACT}

To tackle the subject of emotions in language learning, we look at humour as a facilitating factor in the context of inhibiting emotions in language practice, through a series of examples of pedagogical sequences. We suggest a typology of these sequences implying humour, whether it is initiated by the teacher or by the learner. This typology allows us to shed light on the diversity that is covered by the use of humour in a pedagogical context. In a perspective where language production is considered as language risk-taking, we study the way humour is built into a pedagogical scenario or, on the contrary, appears

* Laboratoire ICAR (UMR 5191), Département d'études du monde anglophone, Université Lumière Lyon 2. 
unexpectedly during a lesson. Humour triggers indeed varied emotions since it can mean, depending on the scenario, pedagogical risk-taking for the teacher, or a risk of losing face for the learner. Our hypothesis is that a non-threatening integration of humour contributes to building a community of trust in the language class and can encourage the emotions that favour language risk-taking.

Dans le contexte d'une analyse de l'apprentissage des langues à la lumière des émotions, nous proposons, à travers une typologie de séquences pédagogiques, d'interroger l'humour comme élément susceptible de favoriser la prise de risque langagière au sein d'une communauté de confiance (Hendry, 2006) dans la classe de langue. Nous faisons l'hypothèse qu'une pédagogie qui laisse la porte ouverte à l'humour bienveillant est à même, dans le cadre de séances de pratique de la langue, de contribuer à minimiser les émotions perturbatrices de la performance linguistique (Wagner \& Urios-Aparisi, 2011). Nous proposons d'aller vers une meilleure lecture de situations pédagogiques convoquant l'humour et une pratique plus informée. En ce sens, nous sommes en accord avec Wagner et Urios-Aparisi (2011) lorsqu'ils se proposent d'améliorer chez les enseignants la prise de conscience de l'importance de l'humour comme outil pédagogique. Ils affirment que des exemples peuvent aider les enseignants à visualiser l'implémentation de la pratique de l'humour. Nous ne cherchons pas ici à faire la preuve par une étude quantitative que l'utilisation de l'humour est plus efficace pour l'apprentissage que d'autres techniques, mais à apporter une contribution à l'articulation des notions d'émotion, d'humour et de prise de risque en pratique de la langue à travers une typologie de séquences pédagogiques. Nous avons sélectionné six exemples tirés de notre pratique pédagogique. Il nous est apparu à posteriori qu'il était intéressant de les regrouper dans le cadre d'une réflexion sur les émotions dans l'apprentissage des langues parce qu'ils illustrent chacun différemment une intervention de l'humour et le rapport à la prise de risque langagière.

Avant d'étudier ces exemples d'activités en pratique de la langue anglaise introduisant l'humour et d'en proposer une typologie, nous présentons d'abord les aspects théoriques qui relient émotions, humour et apprentissage. 


\section{1. Émotions, humour et apprentissage : aspects théoriques}

\section{1. Émotions et apprentissage des langues}

Les chercheurs s'accordent sur l'importance de la prise en compte des aspects émotionnels dans la classe de langue, parce que la «dimension affective atteint tous les aspects de notre existence et de manière très directe ce qui se passe dans la salle de classe, y compris celle de langues étrangères » (Arnold, 2006, p. 408). La salle de classe se prêtant particulièrement à l'activation des émotions (Atienza, 2003), nous nous inscrivons dans une vision holistique de l'enseignement qui prend en compte ces aspects affectifs, couvrant «un large domaine qui comprend les sentiments, les émotions, les croyances, les attitudes et qui conditionne de manière significative notre comportement» (Arnold, 2006, p. 407). Les émotions concernent tout à la fois :

Désir de communiquer avec une personne concrète, confiance en soi, motivation interpersonnelle, motivation groupale, confiance dans ses possibilités en langue cible, attitudes intergroupales, climat intergroupal et personnalité. (Arnold, 2006,p. 411)

Les chercheurs établissent un lien entre émotions et efficacité de l'apprentissage, car «le fait de stimuler les différents facteurs émotionnels, comme l'estime de soi, l'empathie, la motivation, peut faciliter considérablement le processus d'apprentissage d'une langue» (Piccardo, 2007, p. 41).

L'efficacité de l'apprentissage dans une vision holistique qui prend en compte les aspects émotionnels est en partie due à une meilleure motivation (Wagner \& Urios-Aparisi, 2011, p. 402) puisqu'en effet «l'engagement émotionnel dans une activité d'apprentissage intensifie la motivation à effectuer cette activité et en facilite par là même l'accomplissement» (Develotte, 2005, p. 13).

À l'inverse, le «filtre affectif» devient un obstacle s'il n'est pas suffisamment pris en compte, car il :

[...] vient s'interposer entre les données reçues et [1']acquisition par l'apprenant. [...] Ce filtre est, en d'autres termes, un mécanisme de défense psychologique, se traduisant par la peur de se tromper, de sembler ridicule... (Kertesz-Vial, 2000, p. 5) 


\subsection{Humour et apprentissage des langues}

Parmi les aspects affectifs qui traversent la classe de langue et l'apprentissage, nous proposons de nous pencher plus particulièrement sur l'humour. Dans cette partie théorique, nous regardons d'abord ce que l'on entend par humour dans un contexte pédagogique, puis son articulation avec la motivation. Nous exposons comment les notions de prise de risque et de communauté de confiance s'articulent avec les émotions et l'humour dans la perspective d'un apprentissage de langue.

\subsubsection{Humour et incongruité}

L'humour est associé à l'intentionnalité (Baud, 2010), et «le rire ou le sourire, comportement émotionnel» est associé à l'incongruité (Wagner \& Urios-Aparisi, 2011, p. 410), à une «violation des expectatives», «un conflit entre ce qu'on attendait et ce qu'on rencontre effectivement, autrement dit entre les référents dont on dispose et le percept actuel qui ne leur correspond pas» (Bariaud, 1983, p. 25).

Cette incongruité n'est pas l'évidence dans une situation pédagogique. L'humour ne fait pas forcément partie des piliers d'une pragmatique pédagogique. Nous entendons par pragmatique pédagogique les règles d'action dans le contexte pédagogique, qui constituent un ensemble de comportements attendus, que ce soit de la part de l'enseignant ou de l'apprenant. Ainsi, s'il peut être évident pour un apprenant qu'il faut, par exemple, faire le travail demandé par l'enseignant, il ne va pas de soi, si ce n'est pas précisé explicitement, de savoir s'il est autorisé à introduire une dimension humoristique dans une production écrite ou orale.

Dans un contexte interculturel, utiliser cette incongruité en recourant à l'humour, c'est aussi préparer les étudiants aux subtilités de l'intercompréhension (Cazade, 2009).

\subsubsection{Humour et motivation}

L'humour contribue à introduire la variété nécessaire à une meilleure motivation en réduisant la monotonie et l'ennui (Arnold, 2006; Wagner \& Urios-Aparisi, 2011). En effet, l'humour est

l'ennemi d'une pédagogie dogmatique et allié d'un enseignement simple, non incarcéré dans des programmes préétablis et non isolé dans une camisole méthodologique qui s'imposerait à tous, en tout lieu et à tout moment. Il constitue une espèce d'effraction, de surgissement dans la tristesse de la dictature (didactique). (Porcher, 2002, p. 52) 
C'est-à-dire que l'humour s'immisce dans l'interstice que représente la latitude pédagogique dont dispose le praticien. La pédagogie se manifeste et prend corps dans l'espace laissé libre entre le contenu disciplinaire et l'individualité de l'enseignant, éventuellement à travers l'humour.

L'humour aurait le pouvoir de «piquer la curiosité, éveiller l'intérêt, modifier l'attitude du sujet vis-à-vis de l'objet d'étude, permettre un effort soutenu, activer la concentration mentale, recharger l'énergie disponible pour conduire à terme l'ouvrage engagé» (Galisson, 2002, p. 123). Si, comme l'affirme Scrivener (2011), la meilleure manière d'apprendre une langue étrangère est d'être complètement focalisé sur autre chose du point de vue du contenu, le recours aux émotions à travers un humour bienveillant nous semble pouvoir être le vecteur de cette diversion. En effet, ce n'est plus le scénario pédagogique qui est un prétexte à la pratique de la langue, mais la langue qui est l'outil permettant d'accéder à une connivence et à un moment de partage pédagogique au sein de la classe érigée en communauté de confiance (Nadeem, 2012). Il ne s'agit pas d'espérer apprendre sans effort à travers un apprentissage entièrement incident, mais de créer les conditions d'une envie d'apprendre à travers des moments pédagogiques qui invoquent des émotions déshinibitrices. Dans le même ordre d'idées, les débats autour de «questions éthiquement vives» (Rémon, 2012, p. 1) nous semblent relever de cette même diversion. Ils permettent à la langue de devenir le vecteur d'une réflexion citoyenne, le scénario pédagogique n'étant plus seulement un prétexte pour pratiquer la langue.

\subsubsection{Humour, prise de risque et communauté de confiance}

On distingue humour et ironie, car «l'ironie et la dérision sont mal perçues par les jeunes esprits, qu'ils blessent. C'est dire si l'humour dans l'éducation demande à être manié avec prudence et délicatesse » (Fick, 1997, p. 38). Il nous semble que l'humour, utilisé avec bienveillance comme nous le verrons plus loin, peut en effet être classé au rang de «comportement qui confirme» et qui «peut créer une zone de sécurité où l'apprenant n'a pas peur de prendre les risques impliquant la prise de parole en langue étrangère (Arnold, 2006, p. 412-413). Wagner et Urios-Aparisi (2011) parlent d'immédiateté («immediacy») (p. 403) à propos de la proximité dans l'interaction que peut créer le recours à l'humour.

La prise en compte des facteurs affectifs revenant essentiellement à «réduire l'effet des facteurs négatifs et stimuler celui des positifs» 
(Arnold, 2006, p. 411), nous adoptons l'hypothèse selon laquelle l'humour est l'un «des moyens les plus commodes pour faire échec à l'anxiété» (Kertesz-Vial, 2000, p. 6), si l'on suit toutefois des préceptes tels que ceux suggérés par Arnold (2006) pour réduire l'anxiété inhibitrice dans la salle de classe :

- Traiter toujours l'élève avec le plus grand respect et essayer de protéger son moi.

- Donner aux élèves la possibilité d'exprimer leurs préoccupations. Le fait de vérifier que leurs camarades partagent leurs sentiments d'insécurité, qu'ils ne sont pas les seuls à les en ressentir, peut avoir un effet positif. [...]

- Créer une atmosphère de coopération au lieu de concurrence.

- Garder une politique sur la correction d'erreurs qui ne soit pas vécue comme une menace de la part des élèves. (p. 413-414)

Parce qu'il suppose une communauté de confiance, l'humour peut contribuer à lever des obstacles et encourager les apprenants à une plus grande prise de risque langagière (Nadeem, 2012). Nous entendons par prise de risque langagière pour l'apprenant le fait de sortir de sa zone de sécurité en ayant recours à des éléments linguistiques qu'il ne maitrise pas encore parfaitement. Cette «capacité à courir des risques» fait partie des facteurs individuels intervenant dans la réussite de l'apprenant d'une langue étrangère (Arnold, 2006, p. 408).

Cazade (2009) évoque (dans une perspective interculturelle) la prise de risque inhérente au travail sur l'humour comme permettant «en prenant conscience de la difficulté que peut aussi éprouver l'autre, de mieux comprendre la sienne propre, de s'en enrichir, et peut-être de mieux comprendre et accepter l'autre comme un autre soi-même» (p. 35). La prise de risque imposée n'est plus perçue comme telle et permet la production langagière seulement si une communauté de confiance a été établie et l'anxiété réduite «notamment par l'intermédiaire de l'attitude du professeur et de l'atmosphère que celui-ci crée dans la classe» (Arnold, 2006, p. 412).

Dans le contexte de l'apprentissage de la langue maternelle, Charmeux (1997) fait aussi le lien entre humour et sécurité linguistique :

L'humour semble plus nécessaire encore pour la maîtrise de la langue, que pour d'autres maîtrises. On sait, en effet, aujourd'hui, que le sentiment de sécurité linguistique est, par sa présence ou son absence, le premier responsable de la réussite ou de l'échec scolaire. Seul le jeu, et jeu sur le jeu qu'est l'humour, permettent d'installer une relation affective 
positive qui est au cœur de cette sécurité langagière qui caractérise les élèves des milieux favorisés et nourrit leur appétit d'apprendre. (Charmeux, 1997, p. 104)

D'autres chercheurs s'accordent sur les effets de l'humour sur l'anxiété dans l'apprentissage (Anderson \& Arnoult, 1989; Bennett, 2003; Berk, 1996, 2002; Caron, 2002; Cornett, 1986; Garner, 2006; Mahoney, 2000 ; Philaretou, 2006; Stambor, 2006, cités dans Nadeem, 2012) et sur l'apprentissage lui-même (Casper, 1999; Cornett, 1986; Fisher, 1997, cités dans Nadeem, 2012) (Powell \& Anderson, 1985; Askilson, 2005, cités dans Azizifard \& Jalali, 2012). Vaezi et Fallah (2012) interrogent quant à eux la relation entre humour et anxiété du point de vue de la formation et de la pratique enseignante.

Nous prenons ici le parti non pas d'observer les effets de la prise en compte des émotions sur les performances linguistiques des étudiants, mais de regarder à travers différentes séquences pédagogiques comment les conditions d'une prise de risque linguistique et émotionnelle sont réunies à travers l'irruption de l'humour au sein d'une communauté de confiance. Nous établissons une typologie qui prend en compte l'intention initiale de l'enseignant et la prise de risque langagière ou émotionnelle potentielle pour l'apprenant.

\section{Exemples de séquences pédagogiques invoquant I'humour}

Dans une perspective où la dimension affective de l'apprentissage des langues est prise en compte à travers l'humour, nous proposons, à la lumière de plusieurs exemples, et à travers les notions combinées d'émotion, d'humour et de prise de risque, d'étudier quels paramètres sont en jeu dans ces situations pédagogiques et quelle combinaison semble compatible avec la mise en place d'une communauté de confiance, à même de favoriser les émotions positives pour l'apprentissage. Nous avons sélectionné six exemples issus de notre pratique enseignante. Il nous est apparu à posteriori qu'ils avaient en commun d'invoquer l'humour selon des modalités diverses. Ils relèvent de compétences écrites ou orales, en production ou en compréhension. Nous présentons ces exemples en séparant les cas où l'initiative est celle de l'enseignant des cas où l'initiative a été prise par l'étudiant. 


\subsection{Méthodologie}

Au cours de notre activité d'enseignante en pratique de la langue avec des étudiants anglicistes, nous avons pu mettre en œuvre une variété de séquences dont certaines invoquent l'humour de façon constitutive et d'autres où l'humour a été introduit par les apprenants de manière inattendue. Ces séquences se sont déroulées avec des groupes de 30 étudiants anglicistes, en première ou en deuxième année à l'université.

Puisqu'il est apparu à posteriori que ces séquences avaient en commun cette composante humoristique, nous avons souhaité, en adoptant la double posture d'enseignante et de chercheuse, les passer en revue de manière systématique pour y relever, à travers une approche qualitative, la présence de paramètres tels que :

- l'intention de l'enseignant ou de l'apprenant, le caractère volontaire ou imposé de la prise de risque;

- le caractère constitutif ou contingent de l'humour dans le support utilisé ou dans la séquence;

- le caractère menaçant ou non de cette composante humoristique, notamment selon qu'elle s'exerce aux dépens de tous ou de quelques-uns;

- la relation entre humour et prise de risque langagière ou émotionnelle.

Nous présentons ces paramètres sous forme de typologie en conclusion.

Notre choix a porté sur six séquences, impliquant diversement les compétences langagières :

- une séquence de compréhension orale à partir d'une conférence filmée;

- deux séquences de production orale (exposé et improvisation);

- deux séquences de compréhension écrite (d'une fiche d'instructions et de sites web);

- une activité de production écrite sur un blog.

\subsection{Humour introduit par l'enseignant}

Dans les exemples suivants, l'humour est introduit par l'enseignant, par la sélection d'une ressource humoristique en elle-même ou par l'utilisation qui en est faite. 


\subsubsection{Compréhension orale d'une conférence filmée}

Dans le cadre d'un exercice de compréhension orale, les étudiants visionnent une conférence qui porte sur la créativité dans le système éducatif dans laquelle le conférencier fait preuve de beaucoup d'humour. Les étudiants sont divisés en trois groupes : l'un est chargé de relever les mots d'humour, un autre est chargé de relever les idées clés, et un dernier est chargé de relever les exemples donnés par le conférencier. L'objectif en termes d'apprentissage langagier est la pratique de la langue orale en compréhension et en production, car les thématiques abordées permettent ensuite de débattre. L'activité est envisagée sous forme de collecte d'information et non sous forme de test de compréhension.

La prise de risque se situe ici au niveau pédagogique, car l'enseignant choisit d'exploiter l'humour de la ressource au risque de laisser de côté les étudiants dont les moyens linguistiques ne permettraient pas la compréhension de l'humour du conférencier. Même s'il ne s'agit pas d'étudiants débutants, les connaissances supposément attendues par l'enseignant peuvent avoir des effets négatifs même s'il n'y a pas en réalité d'injonction de savoir de sa part. Pour éviter une intervention négative de l'affectif et permettre la mise en place d'une communauté de confiance dans une situation de ce type, le tact pédagogique (Goubet, 2005) mettra en toute simplicité, sans jugement, «les embarras au milieu », «la bêtise au milieu » (Vincent, 2005, p. 15), c'est-à-dire que l'apprenant «doit toujours savoir que si sa réponse peut ne pas être acceptée, lui il l'est toujours» (Arnold, 2006, p. 414). Le travail en groupe, notamment, permet de mettre en place une collaboration entre les apprenants. Un des atouts de l'humour signalé par Arnold (2006) est d'ailleurs sa contribution à la dynamique de groupe par la mise en place d'activités qui «tout en développant des compétences linguistiques, puissent servir à "briser la glace" et à favoriser les relations entre les camarades» (p. 416).

L'humour intervient ici plus au titre de l'incongruité (cf. 1.2.1), de la diversion et de la motivation (cf. 1.2.2) qu'au titre d'une stimulation de la prise de risque langagière. En effet, si l'humour, dans cette séquence, est constitutif de la ressource, il n'est pas dans la consigne elle-même et n'est pas l'objet d'un travail linguistique particulier, mais rend simplement le support plus attrayant. 


\subsubsection{Compréhension écrite $d^{\prime}$ 'une fiche d'instructions piégée}

Dans le cadre d'un exercice de compréhension écrite, une fiche d'instructions ${ }^{1}$ en langue cible est fournie aux étudiants, où il est stipulé qu'ils doivent lire le document jusqu'au bout avant de commencer à répondre. S'ils respectent effectivement cette consigne, ils lisent la dernière ligne qui leur indique que la seule chose à faire est simplement d'écrire son nom à la fin du document dans l'espace prévu à cet effet, sans tenir compte des instructions précédentes. Mais la grande majorité des étudiants ne lit pas les instructions jusqu'au bout et répond à toutes les questions ou commandes.

L'humour réside pour partie dans les instructions qui font se lever les étudiants, taper dans leurs mains, toucher la porte, etc. D'autre part, sur un groupe d'une quarantaine d'étudiants, en général un ou deux connaissent l'exercice, et lisent effectivement les instructions jusqu'au bout avant de commencer. Ceux-là posent leur stylo et ont le sourire aux lèvres.

S'agissant de compréhension écrite, il n'y a pas de prise de risque langagière mais un risque d'incident pédagogique, car la face des étudiants qui «tombent dans le piège» est menacée. La menace est donc constitutive de l'activité. Elle se fait aux dépens de la quasi-totalité du groupe. Le tact pédagogique consistera à inclure plutôt qu'exclure ceux qui se sont laissé prendre. On peut là encore interrompre l'activité et en livrer la clé avant que les émotions négatives ne s'installent. Ici, l'humour participe d'une pédagogie du paradoxe ou de la provocation (De Vecchi \& Carmona-Magnaldi, 2002), relevant de l'incongruité et de la diversion évoquées précédemment.

\subsubsection{Compréhension écrite de sites web canulars}

On propose aux étudiants une liste de sites internet en langue cible dont certains sont des canulars, c'est-à-dire des sites présentés comme sérieux, mais dont l'objectif est soit pédagogique (éducation aux médias) soit de pur divertissement. L'objectif de la séance est la pratique conjointe de la langue (ici la compréhension écrite de sites internet) et des Technologies d'information et de communication pour l'enseignement. Les étudiants doivent parcourir les sites et émettre un juge-

1. <www.brainbashers.com/follow.asp>. 
ment sur leur fiabilité. Une discussion permet de mettre en commun les impressions de chacun (production orale) ${ }^{2}$.

L'humour réside dans l'incongruité des sites proposés, comme par exemple celui qui prétend que les scientifiques ont découvert une pieuvre qui vit dans les arbres. Ce site est d'ailleurs régulièrement pris pour un site «sérieux» par les étudiants. L'aspect d'incongruité et de diversion est donc bien présent une fois de plus avec cet exemple de séquence.

L'humour encourage-t-il dans cette activité la prise de risque langagière à travers des émotions positives? La prise de risque peut être langagière, car il y a une prise de parole lors du débat sur la fiabilité perçue des sites internet qui suit l'exploration des sites, mais elle est aussi affective car la menace potentielle est constitutive de l'activité : les étudiants risquent de perdre la face s'ils jugent fiable un site qui est en fait un canular. Le tact pédagogique permettra de veiller à ce que l'humour ne se fasse pas aux dépens de quelques-uns, mais soit un moment partagé par tous. Ainsi, par exemple, on peut éviter des stratégies comme de faire voter à main levée pour chaque site, et encourager la concertation par équipes.

\subsubsection{Production orale sous contrainte}

Dans le cadre d'un exercice de production orale, on demande aux étudiants de se lever, ce qui est en soi un évènement provocateur dans la classe de langue. Ils ne se rassoient, tour à tour, que s'ils répondent à la consigne, quelle qu'elle soit. Par exemple, dans une séance de travail sur l'improvisation, on prend pour modèle une phrase du film Princess bride: «My name is Inigo Mantoya, you have killed my father, prepare to die! ${ }^{3} \gg$ que l'acteur répète de nombreuses fois. Les étudiants, debout, doivent improviser une phrase sur le même modèle s'ils veulent se rasseoir, par exemple : «My name is Typhaine, you have given me an exam paper, prepare to correct it fast $!^{4} \gg$

2. <www.dhmo.org/>, <http://haggishunt.scotsman.com/>, <www.ovaprima.

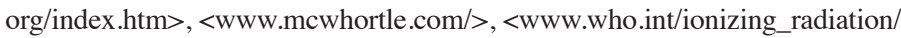

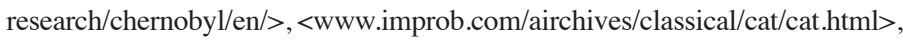
$<$ zapatopi.net/treeoctopus.html>, <www.malepregnancy.com>.

3. «Mon nom est Inigo Mantoya, vous avez tué mon père, préparez-vous à mourir!»

4. «Mon nom est Typhaine. Vous m'avez donné un examen. Préparez-vous à le corriger rapidement!» 
On retrouve ici l'incongruité et la diversion évoquées dans les parties 1.2.1 et 1.2.2, mais cette fois, la prise de risque langagière est bien présente également. Les étudiants sont «physiquement» forcés à cette prise de risque langagière, qui n'est pas volontaire, mais incontournable pour satisfaire la consigne. Cette contrainte sera vue par d'aucuns comme n'ayant rien d'humoristique, la situation pouvant être menaçante pour la face des derniers étudiants qui resteraient debout. Le tact pédagogique doit être ici omniprésent si l'on veut éviter une intervention d'émotions négatives et des «dégâts collatéraux» que l'humour peut occasionner :

J.-M. Robert (2002, p. 114) propose cette distinction : l'humour s'oppose traditionnellement à l'ironie par le fait qu'il ne blesse pas. À l'humour sont associées les notions de protection, de modestie, de tolérance, d'autocritique; à l'ironie, le mépris, l'attaque, la condamnation, le persiflage, etc. (Bougherra, 2007, p. 370)

C'est en mettant l'accent sur l'aspect ludique de l'activité, qui s'apparente par certains côtés au jeu des chaises musicales, que les apprenants se prêtent à l'activité avec plaisir, et que la prise de risque langagière peut devenir volontaire. La bienveillance est de mise de la part de l'enseignant et doit l'être chez les pairs également. On pourra par exemple s'arrêter avant que le nombre d'étudiants debout ne soit trop restreint ou encourager les étudiants assis à «souffler» des réponses pour venir en aide à ceux en position encore inconfortable, et ainsi réintroduire le sourire dans les rangs. Cet inconfort peut-être salvateur dans le cadre d'une prise de risque langagière, si toutefois l'enseignant ne crée pas un climat anxiogène, auquel cas la prise de risque se situe du côté pédagogique avec la possibilité qu'un étudiant refuse de jouer le jeu.

L'humour intervient donc ici au titre de l'incongruité, de la diversion et de la menace potentielle à transformer en contrainte créative.

\subsection{Humour introduit par l'apprenant}

Dans les deux exemples suivants, l'humour n'est pas constitutif de l'activité et il n'y a pas de prise de risque subie par l'apprenant. L'humour est introduit de sa propre initiative.

\subsubsection{Exposé oral}

Également dans le cadre de séances de pratique de la langue et des TICE, la tâche proposée aux étudiants est cette fois une présentation orale en temps limité sur un thème libre, avec exploitation d'un logi- 
ciel de présentation. Les étudiants choisissent une variété de thèmes, et parmi eux une étudiante opte pour le monstre du Loch Ness et introduit un accessoire : elle revêt une blouse blanche pour se déguiser en savant. Sa présentation fait sourire l'auditoire composé de ses pairs.

Les étudiants qui font le choix de l'humour sont peu nombreux, peut-être 3 ou 4 sur un groupe de 25 étudiants, et encore moins nombreux à apporter des accessoires. Ces choix ne sont pas l'évidence en classe de langue. Les étudiants n'en ont pas l'idée, ou ils n'osent pas, ou ils pensent que ce n'est pas autorisé : la pragmatique pédagogique en ce qui concerne l'humour ne va pas de soi.

L'étudiante prend non seulement des risques langagiers en production orale, de surcroit devant un public, mais également le risque de perdre la face si l'effet escompté par sa mise en scène ne se produit pas. Le risque de sortir d'une zone de confort est choisi librement par l'étudiant et est ici synonyme de créativité. On peut observer cette même «mise en danger» de l'étudiant lors de séances de récitation également: lorsque l'on demande aux étudiants de préparer un texte de leur choix à réciter ou lire à haute voix, certains vont jusqu'à choisir un texte à chanter, montrant ainsi que la communauté de confiance est solidement établie. La prise de risque est également du côté de l'enseignant, puisque l'espace d'expression est ouvert : avec une thématique libre, le contrôle pédagogique est moindre.

\subsubsection{Production écrite : rédaction d'un blog}

Toujours dans le cadre de séances de pratique de la langue et des TICE, on demande aux étudiants de créer une brochure en anglais sur support informatique et de commenter les séances chaque semaine en français sur un blog créé pour l'occasion. Les notes publiées sur les blogs sont descriptives pour la plupart. Les traces d'humour sont rares.

Une étudiante pourtant introduit de temps à autre des notes humoristiques :

Je suis en binome avec Laurie (qui me fait planer!!), et le sujet de notre super brochure... L'Australie!! Quel sadisme de nous faire travailler en premier lieu sur Tahiti puis sur un voyage à organiser... Nous pauvres étudiants à la fac, le vendredi de $11 \mathrm{~h} 30$ à $13 \mathrm{~h}$, on a envie que d'une chose, c'est les faire ces voyages !!!!!!! Quelle frustration quand on y travaille là derrière nos ordis !!!!

Cependant, elle hésite car elle n'est pas sure que ce soit autorisé. Une autre étudiante le lui a déconseillé. Elle se résout presque à suivre les conseils de son binôme, et donne même pour titre à une note du 
blog «note sérieuse» (ci-dessous), mais au sein de cette même note, un revirement s'effectue et elle explique qu'elle décide de revenir à sa «liberté d'expression»:

Note serieuse

bon... je vais suivre les conseils de Laurie, 08 avril, nous avons enjolivé notre brochure sur l'Australie et ses bons plans... et puis la liberté d'expression; $\mathrm{j}$ 'y tiens bokou, donc finalement $\mathrm{j}$ 'en reviens à ma methode. Le but de ce blog, n'est il pas de vous montrer ke nous savons nous servir du net, et de ces possibilités?

Ce que l'étudiante entend par «j'en reviens à ma méthode », c'est ne pas rédiger forcément des notes sérieuses mais recourir à l'humour quand elle en ressent le besoin. Comme dans l'exemple précédent (2.3.1), l'humour n'est pas constitutif de l'activité. C'est l'apprenant qui prend le risque d'y recourir. Il fait preuve de créativité dans l'interprétation de la consigne. On retrouve la même incertitude pragmatique que dans le cas précédent. Si l'on ne précise pas explicitement que l'humour est autorisé, les étudiants hésitent à y avoir recours. Cette incertitude dans les attendus pédagogiques permet dans certains cas l'irruption de l'humour, mais on peut supposer que si celui-ci était autorisé dans un contrat didactique plus explicite, les apprenants y auraient recours plus fréquemment.

\section{Discussion}

Au terme de la présentation de ces exemples, nous pouvons récapituler les paramètres nous permettant de mieux les appréhender : l'humour peut être constitutif de la ressource ou incident. Il est tantôt du fait de l'apprenant, tantôt du fait de l'enseignant. Il peut être synonyme de prise de risque pragmatique, langagière ou émotionnelle, mais aussi de divertissement ou de menace. L'inconfort qui en résulte pour les apprenants peut être de degré variable selon que l'humour se fait aux dépens de tous ou de quelques-uns.

Le codage proposé par Wagner et Urios-Aparisi (2011, p. 413-422) nous éclaire sur certains aspects de notre propre typologie. Les auteurs proposent de coder l'humour en contexte pédagogique à la fois en production, en réception, dans ses différentes fonctions et dans son rapport au contenu culturel ou linguistique. Certaines catégories recoupent des paramètres que nous utilisons (tableau 1).

Ainsi, pour ce qui est de la réaction à l'humour, les auteurs proposent la distinction entre réaction de groupe ou réaction individuelle. 
En ce qui concerne la cible de l'occurrence humoristique, les auteurs mentionnent par exemple l'autodérision de la part de l'enseignant, par opposition à de l'humour visant un étudiant en particulier ou encore aucune cible particulière. L'aspect potentiellement menaçant d'une séquence pédagogique recourant à l'humour dépend en effet pour beaucoup de la capacité du pédagogue à mobiliser une implication bienveillante du groupe.

Pour ce qui est du rapport au contenu d'enseignement, l'élément humoristique peut être pertinent ou pas (Nadeem, 2012). Ainsi, dans notre exemple d'exploitation pédagogique d'une conférence filmée (cf. 2.2.1), les aspects humoristiques n'ont pas de lien direct avec un contenu didactique.

Un autre aspect que nous avons également évoqué concerne la source de l'humour : dans la classification de Wagner et Urios-Aparisi (2011, p. 423), elle peut être extérieure ou inhérente au support utilisé.

La typologie ci-dessous (tableau 1) rassemble nos paramètres et permet de mettre en évidence la diversité dans le rapport à la prise de risque que recouvre le recours à l'humour en contexte pédagogique.

\begin{tabular}{|c|c|c|c|c|}
\hline $\begin{array}{l}\text { Humour } \\
\text { introduit } \\
\text { par }\end{array}$ & Activité & $\begin{array}{c}\text { Type } \\
\text { d'humour }\end{array}$ & $\begin{array}{l}\text { Risque pour } \\
\text { l'enseignant }\end{array}$ & Risque pour l'apprenant \\
\hline \multirow{4}{*}{ 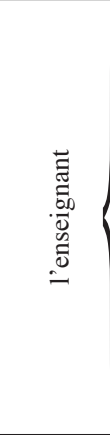 } & $\begin{array}{c}\text { Conférence } \\
\text { humoristique }\end{array}$ & $\begin{array}{l}\text { Incongruité } \\
\text { Diversion }\end{array}$ & \multirow{2}{*}{\begin{tabular}{|c|} 
Si les \\
apprenants \\
pensent qu'il \\
y a une \\
injonction de \\
savoir \\
\end{tabular}} & $\begin{array}{l}\text { Perte de face s'il ne comprend } \\
\text { pas l'humour du conférencier }\end{array}$ \\
\hline & $\begin{array}{l}\text { Sites web } \\
\text { canulars }\end{array}$ & \multirow{3}{*}{$\begin{array}{l}\text { Diversion } \\
\text { Incongruité } \\
\text { Menace }\end{array}$} & & $\begin{array}{l}\text { Perte de face s'il juge sérieux } \\
\text { un site web canular }\end{array}$ \\
\hline & $\begin{array}{l}\text { Improvisation } \\
\text { contrainte }\end{array}$ & & \multirow{2}{*}{$\begin{array}{l}\text { S'il y a refus } \\
\text { de «jouer } \\
\text { le jeu» de } \\
\text { la part d'un } \\
\text { étudiant }\end{array}$} & $\begin{array}{l}\text { - Perte de face s'il est dernier } \\
\text { debout } \\
\text { - Prise de risque langagière } \\
\text { imposée }\end{array}$ \\
\hline & $\begin{array}{l}\text { Instructions } \\
\text { piégées }\end{array}$ & & & $\begin{array}{l}\text { Perte de face s'il ne lit pas les } \\
\text { instructions }\end{array}$ \\
\hline \multirow{2}{*}{ 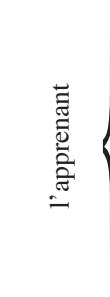 } & Exposé oral & \multirow[t]{2}{*}{ Incongruité } & \multirow[t]{2}{*}{$\begin{array}{l}\text { Perte de } \\
\text { contrôle }\end{array}$} & $\begin{array}{l}\text { - Prise de risque langagière } \\
\text { - Prise de risque émotionnelle } \\
\text { choisie } \\
\text { - Perte de face si l'effet } \\
\text { escompté ne se produit pas }\end{array}$ \\
\hline & $\mathrm{Blog}$ & & & $\begin{array}{l}\text { Risque pragmatique choisi } \\
\text { par rapport aux attendus } \\
\text { pédagogiques }\end{array}$ \\
\hline
\end{tabular}

Tableau 1. - Typologie d'exemples de recours à I'humour en contexte pédagogique. 
Une analyse quantitative permettrait d'étudier chaque combinaison de paramètres en regard de l'efficacité de l'apprentissage et de la différence entre une prise de risque volontaire et une prise de risque imposée, où des émotions différentes sont en jeu. Mais plutôt que de privilégier un type de recours à l'humour, on pourra tendre vers une pédagogie qui se saisisse d'un éventail de possibilités telles que celles présentées ici. Cette diversité même, constitutive de la pédagogie en général, contribue à l'efficacité de l'apprentissage et à la gestion des émotions, notamment en lien avec la motivation, tel qu'évoqué ci-dessus (1.2.2).

\section{Conclusion}

Le risque émotionnel, pédagogique et langagier que constitue le recours à l'humour en apprentissage des langues vaut la peine d'être pris. Par ce biais, on signifie aux apprenants un double message : on transmet un contenu pédagogique et à un deuxième niveau on établit une complicité, on manifeste aux apprenants qu'on les considère capables de comprendre l'humour, qu'on souhaite le partager avec eux, «qu'on a de la confiance en leur capacité à apprendre la langue» (Arnold, 2006, p. 417). Si l'effet loupe de l'humour grossit «toutes les imperfections de conception, d'expression, de maladresses diverses d'un locuteur qui s'essaye à pénétrer de telles finesses et à "faire de l'humour" en langue étrangère », la bienveillance pédagogique de l'enseignant lui permet de «tirer parti» des difficultés de l'apprenant, et de faire de l'humour «un excellent moyen de lutter contre certains blocages, notamment à l'oral» avec «un impact positif sur tout le comportement cognitif et métadidactique de l'apprenant en langues» (Cazade, 2009, p. 1).

En effet, l'humour et l'ouverture d'esprit qu'il implique sont rendus possible par l'habileté de l'enseignant «qui aura su, avec plus ou moins de succès, établir un climat de bonne humeur, de confiance et même de complicité entre les participants» (Cazade, 2009, p. 11). Cette complicité fait écho à la «connivence culturelle» mentionnée par Bougherra (2007) :

Il ne s'agit pas de divertir, de distraire après un travail «sérieux» (grammaire, conjugaison, analyse) mais de mettre le rire au service d'une éducation à la perception sémiolinguistique du quotidien. Les justifications didactiques à la constitution de l'humour en objet à enseigner relèvent du simple constat : la seule maîtrise des composantes fonctionnelles de la langue conduit à la lisière de la communication, à l'exclusion de la connivence culturelle. (p. 367) 
Dans une optique où les émotions sont prises en compte dans l'apprentissage, la création d'une communauté de confiance en classe de langue peut passer par la porte laissée ouverte à l'humour dans un espace pédagogique partagé. Cet espace est conçu dès lors comme le lieu d'expériences communes où les erreurs font partie intégrante du contrat pédagogique (Watson \& Emerson, 1988, cités dans Nadeem, 2012) qui devient contrat de confiance avec des apprenants-citoyens.

\section{RÉFÉRENCES BIBLIOGRAPHIQUES}

Anderson, C. A. \& Arnoult, L. H. (1989). An Examination of Perceived Control, Humor, Irrational Beliefs, and Positive Stress as Moderators of the Relation between Negative Stress and Health. Basic and Applied Social Psychology, 10 (2), 101-117.

ARnold, J. (2006). Comment les facteurs affectifs influencent-ils l'apprentissage d'une langue étrangère? Études de linguistique appliquée, 4 (144), 407-425.

Askildson, L. (2005). Effects of Humor in the Language Classroom: Humor as a Pedagogical Tool in Theory and Practice. Arizona Working Papers in Second Language Acquisition and Teaching, 12, 45-61.

AtienzA, J. L. (2003). L'émergence de l'inconscient dans l'appropriation des langues étrangères. Études de linguistique appliquée, 3 (131), 305328.

Azizifard, F. \& JalaLI, S. (2012). Context and Humor in Teaching Language Functions. Theory and Practice in Language Studies, 2 (6), 1191-1198.

BARIAUD, F. (1983). La genèse de l'humour chez l'enfant. Paris : PUF.

BAUD, M. (2010). Lire pour apprendre, rire pour apprendre? Mémoire de Master 2, Université de Rouen. Disponible sur <www.univ-rouen.fr/ civiic/memoires_DEA/textes/T_baud.pdf>.

Bennett, H. J. (2003). Humor in Medicine. Southern Medical Journal, 96 (12), 1257-1261.

Berk, R. A. (1996). Student Ratings of 10 Strategies for Using Humor in College Teaching. Journal on Excellence in College Teaching, 7 (3), 71-92.

BERK, R. A. (2002). Humor as an Instructional Defibrillator: EvidenceBased Techniques in Teaching and Assessment. Sterling, VA : Stylus Publishing.

Bougherra, T. (2007). Humour et didactique des langues : pour le développement d'une compétence esthético-ludico-référentielle. Études de linguistique appliquée, 3 (147), 365-382. 
Caron, J. E. (2002). From Ethology to Aesthetics: Evolution as a Theoretical Paradigm for Research on Laughter, Humor, and Other Comic Phenomena. Humor, 15 (3), 245-281.

CASPER, R. (1999). Laughter and Humor in the Classroom: Effects on Test Performance. Lincoln : University of Nebraska

Cazade, A. (2009). L'interculturel est-il soluble dans l'humour? Cahiers de l'APLIUT, 28 (2), 24-39. Disponible sur <http://apliut.revues.org/ $1067>$.

Charmeux, É. (1997). Et si l'humour... était le meilleur moyen de lutter contre l'illettrisme et l'échec scolaire en général. Dans H. Lethierry (dir.), Savoir en rire 2, L'humour maître (Didactique et zygomatique) (p. 93-97). Bruxelles : De Boeck Université.

Connett, C. E. (1986). Learning through Laughter: Humor in the Classroom. Bloomington, IN : Phi Delta Kappa Educational Foundation.

Develotte, C. (2006). Le Journal d'étonnement. Lidil, 34. Disponible sur $<$ http://lidil.revues.org/index25.html>.

De Vecchi, G. \& Carmona-Magnaldi, N. (2002). Faire vivre de véritables situations-problèmes ( $2^{\mathrm{e}}$ éd.). Paris : Hachette Éducation.

Dörnyei, Z. \& Malderez, A. (1999). The Role of Group Dynamics in Foreign Language Learning and Teaching. Dans J. Arnold (dir.), Affect in Language Learning (p. 155-169). Cambridge : Cambridge University Press.

FISHER, M. S. (1997). The Effect of Humor on Learning in a Planetarium. Science Education, 81 (6), 703-713.

GaLisson, R. (2002). L'humour au service des valeurs : défi salutaire, ou risque inutile. Le français dans le monde, 122-139.

Garner, R. L. (2006). Humor in Pedagogy: How ha-ha Can Lead to aha! College Teaching, 54 (1), 177-180.

Goubet, J.-F. (2005). Qu'est-ce que le tact pédagogique? Essai d'une définition philosophique. Dans Actes de la journée d'étude du 25 mai 2005 (p. 17-23). IUFM Nord-Pas de Calais. Disponible sur <www.lille.iufm. fr/IMG/pdf/Actes_Tact.pdf $>$.

Hendry, J. (2006). Educating Managers for Post-bureaucracy: The Role of the Humanities. Management Learning, 37 (3), 267-281.

Kertesz-Vial, E. (2000). Filtre affectif, humour et pédagogie de l'italien. Italies, 4, 827-833. Disponible sur <http://italies.revues.org/2385>.

FICK, J.-M. (1997). Une alternative à l'immobilisme. Dans H. Lethierry (dir.), Savoir en rire 2, L'humour maître (Didactique et zygomatique). Bruxelles : De Boeck Université.

Mahoney, D. L. (2000). Is Laughter the Best Medicine or Any Medicine at All? Eye on PsiChi, 4 (3), 18-21. 
Medgyes, P. (2002). Laughing Matters: Humour in the Language Classroom. Cambridge : Cambridge University Press.

NadeEm, M. (2012). Teaching with Humor: A Benevolent Teaching Technique for Second Language Learners in Teacher Education. International Journal of English and Literature (IJEL), 2 (4), 89-96.

NKambou, R., Delozanne E. \& Frasson, C. (2007). Éditorial du numéro spécial Les dimensions émotionnelles de l'interaction dans un EIAH. Revue STICEF, 14. Disponible sur <http://sticef.univ-lemans.fr/num/ vol2007/sticef_2007_editoEmotions.htm>.

Philareatou, A. G. (2006). Learning and Laughing about Gender and Sexuality through Humor: The Woody Allen Case. The Journal of Men's Studies, 14 (2), 133-144.

PicCARdo, E. (2007). «Humain, trop humain». Une approche pour esprits libres : de la nécessité d'une dimension humaniste dans la didactique des langues. Les Cahiers de l'Asdifle, 19, 21-49.

Porcher, L. (2002). L'humour comme le tango : une pensée triste qui se danse... Le français dans le monde, 48-53.

Powell, J. P. \& Anderson, L. W. (1985). Humor and Teaching in Higher Education. Studies in Higher Education, 10, 79-90.

RÉmON, J. (2012). Supports filmiques transversaux en pratique de la langue : document authentique ou authentiquement intéressant? Recherche et pratiques pédagogiques en langues de spécialité - Cahiers de l'APLIUT, 31 (2), 90-102.

Robert, J.-M. (2002). Compréhensible mais pas risible. Le français dans le monde, 114-121.

SCRIVEner, J. (2001). Learning Teaching. Oxford : MacMillan.

Stambor, Z. (2006). How Laughing Leads to Learning. Monitor on Psychology, 37 (6), 62-66.

Underhill, A. (1999). Facilitation in Language Teaching. Dans J. Arnold (dir.), Affect in Language Learning. Cambridge : Cambridge University Press.

Vaezi, S. \& Fallah, N. (2012). Sense of Humor and Emotional Intelligence as Predictors of Stress among EFL Teachers. Journal of Language Teaching and Research, 3 (3), 584-591.

Vincent, H. (2005). Douceur des enseignants? Dans Actes de la journée d'étude du 25 mai 2005 (p. 5-17). IUFM Nord-Pas de Calais. Disponible sur <www.lille.iufm.fr/IMG/pdf/Actes_Tact.pdf $>$.

Wagner, M. \& Urios-Aparisi, E. (2011). The Use of Humor in the Foreign Language Classroom: Funny and Effective? Humor - International Journal of Humor Research, 24 (4), 399-434.

Watson, M. J. \& Emerson, S. (1988). Facilitate Learning with Humour. Journal of Nursing Education, 27, 89. 